\title{
Marked temporal increase in the incidence of type 1 and type 2 diabetes among young adults in Finland
}

\author{
N. Lammi • P. A. Blomstedt • E. Moltchanova • \\ J. G. Eriksson • J. Tuomilehto • M. Karvonen
}

Received: 3 January 2008 /Accepted: 21 January 2008 /Published online: 4 March 2008

(C) Springer-Verlag 2008

To the Editor: Previously we reported data on trends in the incidence of type 1 and type 2 diabetes in Finnish young adults aged 15-39 years during 1992-1996 [1] and found a $7.9 \%$ average annual increase in the incidence of type 2 diabetes. This rising trend needed to be confirmed during a longer period of follow-up, and therefore we extended the study until the year 2001.

The collection of data and the classification of patients with diabetes ascertained during 1992 to 1996 have been described in detail previously [1]. The information on new diagnoses of diabetes in the age group of 15-39 years between 1997 and 2001 were obtained from three nationwide registers in Finland. The first register was the Drug Prescription Register of the Social Insurance Institute, which comprises ATC-DDD codes [2] on all prescriptions since 1994, and for this study all the class A10 drugs (drugs used in diabetes) were reviewed. The second register was the Drug Reimbursement Register of the Social Insurance Institute, which comprises information on persons entitled

\footnotetext{
N. Lammi $(\bowtie) \cdot$ P. A. Blomstedt • E. Moltchanova

J. G. Eriksson · J. Tuomilehto $\cdot$ M. Karvonen

Diabetes Unit, Department of Health Promotion and Chronic

Disease Prevention, National Public Health Institute,

Mannerheimintie 166,

00300 Helsinki, Finland

e-mail: niina.lammi@helsinki.fi

J. G. Eriksson

Department of General Practice and Primary Health Care,

University of Helsinki,

Helsinki, Finland

J. Tuomilehto

Department of Public Health, University of Helsinki,

Helsinki, Finland
}

to free-of-charge medication for diabetes. The entitlement is given in response to an application that includes a detailed medical statement prepared by the treating physician. The third register was the Hospital Discharge Register, maintained by the National Research and Development Centre for Welfare and Health, which includes the treating physician's diagnoses in ICD-10 codes [3].

Data from these registers were linked using the unique personal identification number assigned to every Finnish resident. The date of the first entry in any of these registers was set as the date of the diagnosis of diabetes. The patient had to be recorded in at least two of these registers to be included.

A patient was classified as having type 1 diabetes if two of the following three criteria were fulfilled: (1) an ICD-10 diagnosis referring only to type 1 diabetes; (2) permanent entitlement to free-of-charge medication; (3) only insulin was administered immediately at diagnosis and continued until the end of the year 2004.

A patient was classified as having type 2 diabetes if two of the following three criteria were fulfilled: (1) an ICD-10 diagnosis referring only to type 2 diabetes; (2) permanent or temporary entitlement to free-of-charge medication; (3) only oral glucose-lowering agents had been prescribed.

For patients who could not be reliably classified with this method, the application records for free-of-charge medication were acquired from the Social Insurance Institute and reviewed in order to assign the type of diabetes $(n=791)$. Information on patients with no entitlement to free-of-charge medication $(n=563)$ was reviewed, and most were classified as having gestational diabetes, as expected (persons with gestational diabetes are not entitled to free-of-charge medication). Patients who were impossible to classify as having type 1 or type 2 diabetes were classified as having an undefined type of diabetes. 


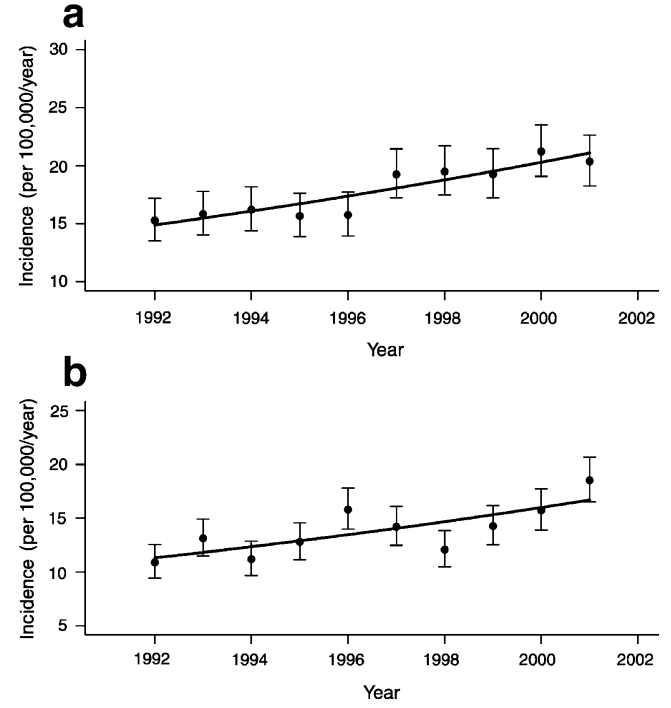

Fig. 1 Observed and estimated annual incidence trends (incidence per 100,000 /year) for type 1 (a) and type 2 (b) diabetes in young adults in Finland. Observed incidence (black circles), 95\% CI for observed incidence (error bars) and estimated trend (black lines)

The total number of patients who fulfilled the inclusion criterion for the years 1997-2001 was 4,062; of these patients, 434 with gestational diabetes and 131 with secondary forms of diabetes were excluded (American Diabetes Association criteria [4]). In total, 3,497 individuals aged 15-39 years were diagnosed with diabetes during 1997-2001. Of these, $48.3 \%(n=1690)$ were classified as having type 1 diabetes and $36.3 \%(n=1271)$ as having type 2 diabetes. For $15.3 \% \quad(n=536)$ the type of diabetes remained undefined. When the data from the years 19921996 [1] were added to the data set, the total number of patients included in the analysis of the annual trend from 1992 to 2001 was 3,078 for type 1 diabetes and 2,392 for type 2 diabetes.

The sex- and age-specific incidences were calculated for 5 -year age groups. Age-standardised annual incidence rates were calculated using the WHO standard European population with equal-sized age groups. The observed cases were assumed to result from a Poisson distribution, and 95\% CIs were estimated using an exact method [5]. The effect of year of diagnosis was assessed using Poisson regression with a logarithmic link function. The statistical analyses were performed using the R software [6].

The results for the 10 year period between 1992 and 2001 confirmed the increasing trend for type 2 diabetes in Finnish young adults. The total age-adjusted incidence of type 2 diabetes was 12.9 (95\% CI 12.4, 13.5) per 100,000/ year. The incidence increased on average by $4.3 \%$ (95\% CI $3.0,5.9 \%)$ per year $(p<0.001$; Fig. 1$)$, and the increase with age was statistically significant $(p<0.001)$. Male predomi- nance was observed in the 35- to 39-year age group. Contrary to the previously observed stable overall incidence of type 1 diabetes between 1992 to 1996 in young adult Finns [1], the analysis combining 10 year data from 1992 to 2001 showed that the incidence of type 1 diabetes increased on average by $3.9 \%$ (95\% CI $2.7,5.3 \%)$ per year $(p<0.001$; Fig. 1). The overall age-adjusted incidence of type 1 diabetes was $18.0(95 \%$ CI $17.4,18.6)$ per 100,000/year. The male/female ratio in the incidence of type 1 diabetes was 1.7. The incidence of undefined diabetes remained stable during this 10 year period.

The increasing trend of $3.9 \%$ per year in the incidence of type 1 diabetes in young adults was similar to the annual $4.2 \%$ increase in the incidence of type 1 diabetes in Finnish children [7]. Thus, the trend previously observed in the child population seems to prevail among the Finnish young adult population also. In addition, the analysis confirms that the incidence of type 2 diabetes in Finnish young adults is increasing markedly. In the first half of this study (19921996) four data sources were utilised, whereas in the second analysis (1997-2001) we did not have the standardised forms filled in by diabetes nurses, which may have caused cases of type 2 diabetes to be dropped from the end of the study period. As this study was register-based, it could not take improved diagnostics into account and patients with type 2 diabetes treated without medication could not be captured by our method. However, the increasing incidence of type 2 diabetes in Finland is supported by the steep increase in the prevalence of the metabolic syndrome [8] in 24-year-old Finns.

The observed increase in the incidence of both type 1 and type 2 diabetes in Finnish young adults is alarming.

Acknowledgements This work was funded by the National Institutes of Health (grant DK062374-01A1) and the Academy of Finland (grants 207008 and 214111).

Duality of interest The authors declare that there is no duality of interest associated with this manuscript.

\section{References}

1. Lammi N, Taskinen O, Moltchanova E et al (2007) A high incidence of type 1 diabetes and an alarming increase in the incidence of type 2 diabetes among young adults in Finland during 1992 to 1996. Diabetologia 50:1393-1400

2. World Health Organization. ATC/DDD index 2007. Available from http://www.whocc.no/atcddd/indexdatabase/, accessed 18 December 2007

3. World Health Organization. International Classification of Diseases. Available from http://www.who.int/classifications/icd/en/, accessed 18 December 2007 
4. American Diabetes Association (2006) Diagnosis and classification of diabetes mellitus. Diabetes Care 29(Suppl 1):S43-S48

5. Aragon TR (2007) Package for epidemiologic data and graphics. Available from http://www.epitools.net, accessed 18 December 2007

6. R Development Core Team (2007) R: a language and environment for statistical computing. Available from http://www.R-project.org, accessed 18 December 2007
7. The DiaMond Study Group (2006) Incidence and trends of childhood type 1 diabetes worldwide 1990-1999. Diabet Med 23: 857-866

8. Mattsson N, Ronnemaa T, Juonala M, Viikari JS, Raitakari OT (2007) The prevalence of the metabolic syndrome in young adults. The cardiovascular risk in young Finns study. J Intern Med 261:1 $59-169$ 